\title{
El nexo entre cambio climático y energía renovable en el Mercosur. Un análisis comparativo de las legislaciones de Argentina y Brasil ${ }^{* * *}$
}

\author{
The climate change-renewable \\ energy nexus in Mercosur. \\ A comparative analysis of Argentina's \\ and Brazil's legislations
}

RESUMEN

El sector energético es uno de los principales contribuyentes al cambio climático debido a la combustión de hidrocarburos, por lo que la energía renovable se presenta como una alternativa para promover el desarrollo sustentable. En ese sentido, aplicando una metodología comparativa, este artículo analiza las regulaciones sobre cambio climático y energía renovable en Argentina y Brasil, considerados los dos Estados política y económicamente más fuertes del Mercosur, los cuales están especialmente afectados por el cambio climático. En esa lógica, se examina si los Estados priorizan el desarrollo sustentable o la seguridad energética, de qué manera las políticas del Mercosur influencian

Abogada por la Universidad Nacional de Córdoba, Argentina. Doctora en Estudios Globales (PhD in Global Studies) por la Universidad de Leipzig, Alemania. Funcionaria judicial de la Provincia de Córdoba. Docente de Derecho Internacional Privado y adscrita a la Cátedra de Derecho Internacional Público de la Universidad Nacional de Córdoba (Argentina). Contacto: laura_casola@hotmail.com

Licenciado en Ciencia Política (Especialización en Relaciones Internacionales) por la Universidad de Leipzig, Alemania; doctoren Estudios Globales (PhD in Global Studies) por la Universidad de Leipzig, Alemania. Profesor titular de la cátedra de Teoría de las Relaciones Internacionales (UCC). Director del proyecto de investigación "Difusión de Políticas en el Mercosur. Entendiendo la Adopción de Políticas sobre Energía Renovable y Cambio Climático en Argentina y Brasil" de la UCC. Director del Programa Argentino-Alemán de Cooperación Académica de la Facultad de Ciencia Política y Relaciones Internacionales (UCC) (Argentina). Contacto: afreier07@gmail.co

**** Recibido el 23 de mayo de 2017, aprobado el 15 de octubre de 2017.

Para citar el artículo: Casola, L. y Freier, A. El nexo entre cambio climático y energía renovable en el Mercosur. Un análisis comparativo de las legislaciones de Argentina y Brasil. Derecho del Estado n. ${ }^{\circ} 40$, Universidad Externado de Colombia, enero-junio de 2018, pp. 153-179. DOI: https://doi.org/10.18601/01229893.n40.07 
las decisiones nacionales, y si existen estándares comunes que posibiliten una futura integración regional en la materia.

PALABRAS CLAVE

Cambio climático, energía renovable, desarrollo sustentable, seguridad energética, Mercosur.

\section{ABSTRACT}

The energy sector is one of the biggest contributors to climate change through the burning of fossil fuels. Therefore, renewable energy represents an alternative to promote sustainable development. In this line, applying a comparative approach, this article analyses the climate change and renewable energy regulations in Argentina and Brazil, as Mercosur's economically and politically most powerful nations, which have shown to be particularly affected by climate change. As a result, this work will examine if domestic regulations give priority to sustainable development or to energy security, in which way Mercosur policies influence national decisions, and if common standards leading to a future regional integration in this field can be observed between the two countries.

KEYWORDS

Climate change, renewable energy, sustainable development, energy security, Mercosur.

SUMARIO

Introducción. 1. El contexto normativo del Mercosur. 1.1. El concepto de región. 1.2. La regulación de las energías renovables a nivel regional. 1.3. El rol de las energías renovables en la lucha regional contra el cambio climático. 2. Escenarios nacionales: regulación del cambio climático y de las energías renovables. 3. La relación entre medio ambiente y energía en la República Argentina. 3.1. La importancia de las fuentes renovables en la política energética argentina. 4. La relación entre medio ambiente y energía en la República Federativa de Brasil. 4.1. La importancia de las fuentes renovables en la política energética brasilera. Reflexiones finales. Referencias. 


\section{INTRODUCCIÓN}

El cambio climático, entendido como la alteración de la composición de la atmósfera y su consecuente impacto negativo en la estructura y el funcionamiento de los ecosistemas naturales, los sistemas socioeconómicos, la salud o el bienestar, derivados de la acción humana ${ }^{1}$, no es un proceso reciente. Por el contrario, el origen de este fenómeno se remonta al siglo XVIII, a partir de la Revolución Industrial que, como contracara del desarrollo, trajo también aparejado el deterioro del medio ambiente y la degradación del paisaje. Sin embargo, en las últimas décadas se ha advertido la intensificación de sus efectos, con motivo de la creciente presión humana sobre el ambiente.

En términos simples, el cambio climático es una consecuencia del aumento de la temperatura del medio ambiente terrestre, lo cual provoca consecuencias adversas tales como el derretimiento de glaciares, el aumento del nivel del mar, sequías y la extinción de flora y fauna. En relación con ello, el avance de la ciencia ha podido determinar que la principal causa del calentamiento global es la presencia en la atmósfera de ciertos gases -denominados gases de efecto invernadero (GEIS) - que retienen el calor emitido por la superficie terrestre (Secretaría de Energía de la Nación, 2004, 8). El dióxido de carbono $\left(\mathrm{CO}_{2}\right)$ es uno de los que se encuentra en mayor concentración en la atmósfera y tiene su fuente primordial en la quema de fósiles (ibíd., 8), derivada fundamentalmente de la actividad del sector energético.

De la última aseveración se desprenden dos ideas que deben orientar a los gobiernos y a los actores privados en sus acciones para frenar el cambio climático: la necesidad de reducir la emisión de gases de efecto invernadero y la necesidad de transformar la industria energética, basada principalmente en combustibles fósiles.

En cuanto al primer aspecto, la Convención Marco de las Naciones Unidas sobre Cambio Climático, de 1992, representó un gran paso hacia adelante en materia de protección medioambiental y seguridad humana, porque implicó el reconocimiento internacional de la existencia y gravedad del cambio climático. Sin embargo, su carácter de "convenio marco" demandó la aprobación del Protocolo de Kioto, en 1997, focalizado específicamente en lograr la reducción de la emisión de GEIs a través de medidas jurídicamente vinculantes.

En cuanto al segundo aspecto, a partir del reconocimiento de la existencia del cambio climático y sus efectos adversos, muchos países del mundo comenzaron a pensar en la necesidad de modificar sus matrices energéticas, sustituyendo fósiles por renovables, a fin de reducir las emisiones de GEIS y contribuir al desarrollo sustentable. Sin embargo, las inversiones en energías

1 De acuerdo a lo establecido en el artículo 1, párrafo 2, de la Convención Marco de las Naciones Unidas sobre el Cambio Climático. Disponible en: https://unfccc.int/resource/docs/ convkp/convsp.pdf Consulta: 01/8/16. 
renovables son aún insignificantes en comparación con las inversiones anuales realizadas en el desarrollo de nuevas reservas de combustibles fósiles, algo absolutamente incompatible con la protección del clima global (Secretaría de Energía de la Nación, 2004, 5).

La región del Mercosur, por su gran riqueza en recursos naturales, es una de las áreas más afectadas por el cambio climático (Ministério da Ciência, Tecnología e Inovação, 2016, pp. 24 ss.; Secretaría de Ambiente y Desarrollo Sustentable de la Nación, 2015, 10 ss.). Además de ello, cuenta con extensas reservas de hidrocarburos que son masivamente explotados para abastecer la industria energética (Freier, 2016a), de modo tal que la región es, al mismo tiempo, emisora de GEIs y perjudicada por sus efectos.

A continuación se analiza la relación de la política de cambio climático con la promoción de las energías renovables, en Argentina y Brasil, así como la influencia que en ellos ejerce el contexto regional del Mercosur. Para ese fin, se busca respuesta a los siguientes interrogantes: ¿en qué medida la política energética es considerada una herramienta contra el cambio climático? ¿Los Estados priorizan el desarrollo sustentable o la seguridad energética? ¿En qué grado el Mercosur funciona como marco orientador de la acción colectiva de los Estados, en esta área? ¿Existen estándares comunes que posibiliten una futura integración regional en materia de energías renovables?

\section{EL CONTEXTO NORMATIVO DEL MERCOSUR}

La protección medioambiental ha sido, desde el inicio, un tema central para el Mercosur, ya que en el Tratado de Asunción se dejó claramente establecido que el desarrollo económico con justicia social debe alcanzarse "a través del aprovechamiento más eficaz de los recursos disponibles y de la preservación del medio ambiente" ${ }^{2}$. De igual modo, el bloque también ha dedicado parte de su agenda de trabajo al estudio de la situación energética regional, para procurar el abastecimiento interno. A continuación se analiza en qué medida la política energética ha sido utilizada para lograr el desarrollo sustentable de la región, y su influencia, potencial o efectiva, en las regulaciones nacionales.

\subsection{El concepto de región}

Como consideración previa al análisis propuesto, vale señalar que el concepto de región utilizado en el artículo debe ser entendido de forma multidimensional, con base en la idea de una geografía política consistente, primordialmente, en Estados independientes y soberanos que coexisten con otros actores que

2 De conformidad con lo establecido en el segundo párrafo del Preámbulo del Tratado de Asunción, de 1991, constitutivo del Mercado Común del Sur (Mercosur), disponible en: http:// www.mercosur.int/innovaportal/file/719/1/CMC_1991_TRATADO_ES_Asuncion.pdf 
influencian las relaciones a nivel internacional. En tal sentido, la región implica una superposición de dos tipos diferentes de relaciones: por un lado, los procesos de decisión política gubernamentales o domésticos, por otro, las relaciones que surgen a nivel intergubernamental. En esa lógica, el concepto aplicado asume que los Estados son los principales actores del Mercosur, pero también reconoce que existe un nivel regional institucionalizado capaz de influenciar las decisiones de los gobiernos nacionales (Freier, 2016a).

Siguiendo esa línea, si bien el Mercosur se compone actualmente de cinco Estados, el análisis de la cooperación energética se enfoca aquí, en particular, en la relación entre Argentina y Brasil como subconjunto regional, debido a que el binomio representa un caso muy interesante en sí mismo, en atención a que se trata de los dos socios fundadores del bloque con mayor tamaño y poder económico, con una gran riqueza en recursos naturales renovables y no renovables, con diferentes ideas sobre política energética y con capacidad suficiente para influir en la acción colectiva.

\subsection{La regulación de las energías renovables a nivel regional}

La promoción de las energías limpias, aunque con importancia fluctuante a través del tiempo, ha estado siempre presente en la agenda regional del Mercosur, tal como lo evidencia la evolución normativa e institucional en la materia. En 1993, al poco tiempo de iniciar su actividad institucional, el Grupo Mercado Común aprobó las Directrices de Políticas Energéticas en el Mercosur (Mercosur/GMC/Res. 57/93), entre las que se mencionan la promoción de la producción y uso de energía renovable con bases económicas y ambientalmente sustentables (directriz n. ${ }^{\circ}$ 9) y la armonización de la legislación ambiental y el establecimiento de estructuras que permitan mitigar los impactos sobre el medio ambiente resultantes de la producción, transporte, almacenamiento y uso de los energéticos (directriz n. ${ }^{\circ} 10$ ). Dicho documento muestra que desde los albores del proceso de integración, las energías renovables fueron tenidas en cuenta para lograr el desarrollo regional sustentable.

Siguiendo esa línea, en el año 2000, a través de la Decisión 60/00, se creó la Reunión de Ministros de Minas y Energía del Mercosur (RMME), encargada de proponer medidas tendientes a la coordinación y la cooperación en dichas áreas. Luego, en 2005 se creó, como órgano técnico, el Subgrupo de Trabajo n. 9 "Energía" (SGT 9), mediante Decisión n. ${ }^{\circ}$ 07/05 del CMC, y si bien desde 2011 no han tenido lugar nuevas reuniones en su seno ${ }^{3}$, en sus siete años

3 El principal problema que suscitó la suspensión de actividades fue la falta de acuerdo entre los Estados, respecto al tema del tránsito de energía dentro del Mercosur. En aquella época, Paraguay planteó la libre disponibilidad para poder colocar sus excedentes hidroeléctricos en terceros Estados tales como Uruguay y Chile. Pero Argentina -considerado, por su posición geográfica, un país de tránsito- no estuvo de acuerdo y quiso imponer condiciones que no fueron aceptadas por Paraguay. En relación con esa desavenencia, Brasil, Uruguay y Chile no opinaron, 
de actividad se han emprendido algunos significativos proyectos que han buscado apuntalar a las energías renovables dentro de la región.

Así, en diciembre de 2005 se firmó el Acuerdo Marco de Complementación Energética entre los Estados parte y asociados, en el cual se mencionan los recursos renovables y las energías alternativas como una de las potenciales áreas de cooperación (art. 6), no obstante lo cual el acuerdo no ha sido ratificado, a la fecha, por ningún Estado.

Posteriormente, en 2009, el Consejo del Mercado Común -a instancia del SGT 9- aprobó las Directrices de Fuentes Renovables de Energía en el Ámbito del Mercosur (Mercosur/CMC/Rec. 02/09), que declaran de interés la promoción de fuentes renovables (solar, eólica, hidroeléctrica de pequeño tamaño, geotérmica, undimotriz, mareomotriz y biomasa) para generación eléctrica y de calor en forma sustentable. Asimismo, las Directrices recomiendan incrementar gradualmente la participación de esas fuentes en las matrices nacionales, promover el intercambio de información, analizar oportunidades de cooperación en zonas de frontera y adoptar medidas para promover la fabricación nacional de equipamientos para energías renovables. También en 2009, el SGT 9 trabajó en una propuesta de cooperación con la ex GTZ (Alemania) en un proyecto denominado "Fortalecimiento de la Integración Regional, de la participación y del combate al cambio climático en el Mercosur, sobre la base del fomento de la eficiencia energética y el uso de energías renovables" (SGT 9 Energía/Acta 01/09 - Anexo VII), el cual nunca llegó a ejecutarse debido a diferencias políticas ${ }^{4}$.

En 2010, un representante de la Comisión de Cooperación Técnica (сСт) entre el Mercosur y Alemania informó respecto de una propuesta de cooperación de aquel país sobre eficiencia energética y energías renovables, y se presentó el proyecto de un memorándum de cooperación entre el Mercosur y Alemania, destinado a brindar al primero asesoramiento técnico, capacitación de recursos humanos y posibilitar el intercambio de expertos para la promoción de energías renovables en la región, el cual nunca fue aprobado.

Finalmente, cabe señalar que, en la misma línea, el SGT 9 ha buscado promover la cooperación regional en materia de energía renovable mediante seminarios y jornadas de capacitación, con el propósito de orientar la acción colectiva a través de la redefinición de las ideas nacionales ${ }^{5}$.

y no se llegó a acuerdo alguno. Después de Uruguay, la presidencia pro tempore le correspondió a Argentina, y debido a que no se había podido destrabar el conflicto con Paraguay, se decidió no convocar a las reuniones del SGT 9. Del mismo modo, cuando los demás países fueron asumiendo la presidencia pro tempore, tampoco reactivaron las reuniones (información provista por Guillermo Koutoudjian y María Soledad Álvarez, representantes nacionales en el SGT 9).

4 Información provista por Guillermo Koutoudjian y María Soledad Álvarez, representantes nacionales en el SGT 9.

5 A modo de ejemplo, se puede mencionar el seminario "Las energías renovables en el ámbito del Mercosur, sus Estados Asociados y en el ámbito internacional: su dimensión estraté- 
En síntesis, de lo reseñado hasta aquí se puede observar claramente el interés de la institución regional en promover la progresiva recomposición de las matrices energéticas nacionales, algunas de ellas basadas en altos porcentajes de fuentes no renovables (Freier, 2016a), para lo cual el Mercosur ha oficiado de mediador entre voluntades políticas, en muchos aspectos, disímiles.

\subsection{El rol de las energías renovables en la lucha regional contra el cambio climático}

Tomando como punto de partida la afirmación del interés del bloque en la promoción de las energías verdes, cabe preguntar si dicha política forma parte de la estrategia regional de lucha contra el cambio climático. La dinámica entre ambas áreas puede establecerse de manera que exista entre ellas una interrelación significativa, traducida en la procura de seguridad energética sustentable $^{6}$, o bien puede traducirse en su funcionamiento como compartimentos estancos, en virtud de lo cual la política energética busca lograr la seguridad energética independientemente de la responsabilidad medioambiental. En esa línea se analiza a continuación la política ambiental del Mercosur, a fin de establecer si la promoción de las energías limpias es solo complementaria de la industria tradicional o si, por el contrario, se busca la paralela reducción de la energía fósil, como mecanismo para reducir las emisiones de dióxido de carbono y contener los efectos del cambio climático.

Desde una perspectiva puramente institucional, corresponde señalar que los órganos principales del área de medio ambiente son, por un lado, la Reunión de Ministros de Medio Ambiente del Mercosur (Mercosur/CMc/Dec. 19/03), que es el órgano político, y, por el otro lado, el Sub Grupo de Trabajo n. ${ }^{\circ} 6$ "Medio Ambiente" (Mercosur/Gmc/Res. 20/95), que es el órgano técnico. Si bien de forma directa no ha habido ninguna actividad vinculada al uso de las fuentes alternativas como estrategia para la reducción de GEIs, se han llevado adelante trabajos que han promovido el acercamiento de las áreas de medio ambiente y de energía, a fin de que trabajen en forma coordinada, sumando esfuerzos, para promover el desarrollo sustentable.

gica, productiva, ambiental y económica". Disponible en: http://www.mercosur.int/innovaportal/ file/1398/1/Seminario_EERR_Version_Final.pdf

6 El concepto de seguridad energética sustentable se compone de dos nociones básicas: seguridad energética y desarrollo sustentable. La primera implica el acceso, por parte de los Estados, a la energía de manera segura, confiable y económica (Yergin, 2006), en tanto la segundo hace referencia al desarrollo conjunto y equilibrado del medio ambiente, la sociedad y la economía, por lo que necesita ser complementada con la idea de la sustentabilidad, para minimizar los efectos de la degradación medioambiental y el cambio climático (ElKIND, 2009).

7 Este órgano técnico surgió en 1992 como Reunión Especializada en Medio Ambiente (Mercosur/GMC/Res. n. ${ }^{\circ}$ 22/92), y fue posteriormente jerarquizada, transformándose en 1995 en el Sub Grupo de Trabajo n. ${ }^{\circ} 6$. 
Así, acompañando a la directriz n. ${ }^{\circ} 10$ sobre Políticas Energéticas en el Mercosur -ya mencionada en el punto anterior-, la cual recomienda armonizar las legislaciones para mitigar los efectos de la producción, transporte, almacenamiento y uso de la energía, la Resolución GMC 38/95 encomendó al SGT 6 que coordine la implementación de las propuestas en materia ambiental de los demás subgrupos de trabajo (Silva Gilli, 2004), promoviendo el contacto entre ambas áreas.

Luego, en el año 2001 se aprobó, a nivel regional, el Acuerdo Marco sobre Medio Ambiente del Mercosur (Mercosur/CMC/Dec. 02/01), que entró en vigencia en los Estados parte en el año 2004, el cual tiene como objetivo el desarrollo sustentable y la protección del medio ambiente, mediante la articulación de las dimensiones económicas, sociales y ambientales, contribuyendo a una mejor calidad del ambiente y de la vida de la población (Secretaría Mercosur, 2006, 6).

Siguiendo esa línea, a mediados de 2004, la 1. a Reunión de Ministros de Medio Ambiente (RMMA) aprobó la Declaración sobre Cambio Climático, en la cual los firmantes reconocen los efectos adversos de dicho fenómeno y la consiguiente necesidad de reducir las emisiones de gases de efecto invernadero, la importancia de la pronta entrada en vigor del Protocolo de Kioto, la necesidad de poner en marcha el Mecanismo de Desarrollo Limpio, y la importancia para la humanidad de buscar formas de adaptación al cambio climático, atentendiendo a que algunos de sus efectos son irreversibles.

En el mismo sentido, durante la 10. ${ }^{\mathrm{a}}$ RMMA, realizada en 2009, se analizó -aunque sin resultados concretos- una "Propuesta de Lineamientos" para un proyecto de cooperación de adaptación al cambio climático en el Mercosur, tendiente a la elaboración de un análisis de vulnerabilidad regional e impactos del cambio climático, que sirviera como base para el posterior desarrollo de estrategias coordinadas de adaptación a tal fenómeno. Asimismo, “[m]ás allá de las posibles estrategias coordinadas de adaptación, se apuntó a lograr cooperación financiera internacional, transferencia tecnológica y creación de capacidades de acuerdo a las necesidades de los países de la región" (Stuhldreher, 2012, 196).

No obstante los desarrollos normativos regionales en materia ambiental, hay que reconocer que ni el Acuerdo Marco, ni la Declaración Ministerial, ni la Propuesta de Lineamientos mencionan, en particular, el tema de las energías renovables como herramientas de lucha contra el cambio climático. Si bien dichos documentos reconocen que la protección del medio ambiente es un tema de interés común -lo cual constituye, sin duda, un contexto propicio para la implementación de las nuevas formas de energía limpia a nivel regional-, no involucran expresamente la política energética.

Sin embargo, como un evento significativo hacia una posible convergencia, cabe destacar que durante la 2. ${ }^{\mathrm{a}}$ RMMA, que tuvo lugar en 2005, la Presidencia Pro Témpore de Uruguay (PPTU) presentó una propuesta de energías renovables 
orientada a la concertación de acciones entre las áreas de medio ambiente y energía. En dicho documento, la RMMA reconoce que el tema vinculado al desarrollo de las fuentes de energía renovable ha ganado un importante lugar en las agendas internacionales, debido a que tiene la capacidad de contribuir tanto a la mitigación de los efectos del cambio climático como a la creación de nuevas oportunidades para el sector productivo aprovechando los recursos propios de los Estados de la región (Anexo IX).

Así, de lo analizado se desprende que aun cuando los acuerdos buscados a nivel del Mercosur en muchos casos no se han concretado y en otras oportunidades no han tenido trascendencia normativa en el ámbito doméstico, la institución regional, sin embargo, representa un importante foro de comunicación y negociación en el marco de orientaciones políticas y comerciales divergentes. De igual manera, a pesar de que el proyecto de interacción entre el SGT 9 y el SGT 6 no ha dado todavía resultados, es importante observar que en ambos grupos existe conciencia sobre la vinculación de las dos áreas para lograr el objetivo del desarrollo sustentable de la región.

\section{ESCENARIOS NACIONALES: REGULACIÓN DEL CAMBIO CLIMÁTICO Y DE LAS ENERGÍAS RENOVABLES}

Como se mencionó sucintamente en la introducción, debido a la gran extensión territorial y variedad de climas y recursos naturales que poseen Argentina y Brasil, el cambio climático los afecta en gran medida y de diferente forma en las distintas regiones internas y en los variados sectores de sus economías. Es por ello que, reconociendo los problemas derivados de tal fenómeno global, ambos países asumieron compromisos nacionales e internacionales para contrarrestarlo.

En cuanto a su participación en el movimiento internacional contra el cambio climático, tanto Argentina como Brasil ratificaron en 1994 la Convención Marco de las Naciones Unidas sobre el Cambio Climático (CMNUCC). Dicho instrumento tiene la virtud de ser el primer documento internacional en el cual los numerosos Estados signatarios reconocieron formalmente la existencia del fenómeno de cambio climático y la necesidad de adoptar medidas coordinadas para contrarrestar sus efectos adversos ${ }^{8}$.

Sin embargo, la Convención es genérica y no contiene medidas de acción concretas (Urrutia Silva, 2010, 603-605). Así fue que, luego de acordar que la lucha contra el cambio climático debía focalizarse primordialmente en la reducción de emisiones de GEIs, la comunidad internacional aprobó en 1997 el Protocolo de Kioto -vinculado a la CMNUCC- que contiene mecanismos

8 De acuerdo a lo informado en la página web de la cMNUCC: http://unfecc.int/portal_espanol/informacion_basica/la_convencion/items/6196.php 
obligatorios pero flexibles ${ }^{9}$, en tanto las partes pueden, de acuerdo a sus circunstancias particulares, optar por el que les resulte menos oneroso y más conveniente a sus intereses nacionales.

Argentina ratificó el Protocolo en 2001 y Brasil hizo lo propio en 2002, de modo que ambos países quedaron obligados a cumplir con los compromisos allí contenidos, en carácter de partes no-Anexo $\mathrm{I}^{10}$. Esto último significa que no han asumido un compromiso cuantificado de reducción de las emisiones de GEIs, es decir, no se han comprometido a lograr la reducción de un porcentaje determinado (como sí lo han hecho los países del Anexo I), sino que deben realizar esfuerzos para formular, aplicar, publicar y actualizar regularmente programas nacionales y regionales, que contengan medidas orientadas a mitigar el cambio climático (Protocolo de Kioto, art. 10).

En ese contexto de compromiso nacional e internacional, el sector energético, en ambos Estados, resulta un área clave para lograr el fin propuesto. Si bien los dos países cuentan tanto con gran variedad de recursos renovables como con amplias reservas de hidrocarburos, enfrentan el desafío de utilizar sus óptimas condiciones naturales para generar energía renovable y logar el abastecimiento energético en un contexto dominado por la energía de origen fósil. Esto es así porque "el petróleo y el gas siguen siendo un símbolo de orgullo e independencia nacional, y su explotación es vista como necesaria para el crecimiento económico, por lo que los procesos de decisión política muestran tendencias contradictorias" (Freier, 2016b, 177).

A continuación se analiza la vinculación entre la política medioambiental y la política energética, implementadas por ambos Estados, a fin de observar en qué medida se promueve el uso de energías renovables y la gradual sustitución de los fósiles como estrategia contra el cambio climático.

9 Dichos mecanismos son: el comercio de los derechos de emisión, que permite que los países que tengan unidades de emisión de sobra (emisiones que tienen permitidas pero a las que no llegan) vendan ese exceso de capacidad a países que sobrepasan sus metas; el mecanismo para un desarrollo limpio (MDL), que permite que un país que haya asumido el compromiso de reducir o limitar las emisiones pongan en práctica proyectos de reducción de las emisiones en países en desarrollo. A través de tales proyectos se pueden conseguir créditos por reducciones certificadas de las emisiones, que cuenta para el cumplimiento de sus metas; finalmente, el mecanismo de aplicación conjunta permite que un país que se haya comprometido a reducir o limitar sus emisiones gane unidades de reducción de las emisiones generadas en un proyecto de reducción o eliminación de las emisiones de otra parte del Anexo B, lo que cuenta para el logro de sus metas (ver información contenida en la página web de la CMNUCC: http://unfecc.int/ portal_espanol/informacion_basica/protocolo_de_Kioto/organizacion/mecanismos/items/6219. php).

10 Las partes listadas en el Anexo I de la CMNUCC y las partes listadas en el Anexo B del Protocolo de Kioto son los países desarrollados y los que se encuentran en proceso de transición a una economía de mercado, los cuales han asumido un compromiso cuantificado de reducción de emisiones de gases de efecto invernadero. 


\section{LA RELACIÓN ENTRE MEDIO AMBIENTE Y ENERGÍA EN LA REPÚBLICA ARGENTINA}

Antes de ingresar al análisis del marco legal vigente corresponde realizar una digresión vinculada al control de los recursos naturales. Argentina es un país federal (art. $1 \mathrm{CN}$ ), lo que significa que posee dos niveles de gobierno: nacional y provincial, a los que se suma el régimen de la Ciudad Autónoma de Buenos Aires. En esa línea, las provincias son preexistentes a la Nación y ejercen todos los poderes que no han sido expresamente delegados (art. $121 \mathrm{CN})$.

Propiamente en materia ambiental y de recursos naturales, cabe efectuar la distinción entre dominio y jurisdicción (Nonna, Waitzman y Dentone, 2011). En esa lógica, la Constitución Nacional, en su artículo 124, establece claramente que el dominio originario de los recursos naturales les corresponde a las provincias. Sin embargo, en materia de jurisdicción sobre el ambiente -y por ende, también con relación a los recursos naturales que forman parte de él- la línea divisoria no resulta tan tajante. Por el contrario, a partir de la reforma constitucional de 1994, la protección medioambiental es competencia concurrente de las dos esferas de gobierno (art. $41 \mathrm{CN}$ ).

A pesar de que las provincias son las dueñas originarias de los recursos naturales, la protección del medio ambiente traspasa las barreras jurisdiccionales locales. Ello se debe a que los fenómenos ambientales se caracterizan por su movilidad y por lo tanto generan problemáticas ambientales jurisdiccionalmente divisibles (Nonna, Waitzman y Dentone, 2011). En este entendimiento, en 1990 se creó el Consejo Federal del Medio Ambiente (COFEMA) como espacio de concertación para abordar los problemas y las soluciones del medio ambiente de la República Argentina, y en 1993 se suscribió el Pacto Federal Ambiental, con el fin de establecer acuerdos marco entre las provincias, y entre estas y la Nación, para promover políticas ambientales adecuadas. Ese ámbito de concertación federal es el espacio en el cual comenzaron a desarrollarse las políticas ambiental y de cambio climático en Argentina.

La reforma constitucional de 1994 estableció que "[c]orresponde a la Nación dictar las normas que contengan los presupuestos mínimos de protección, y a las provincias, las necesarias para complementarlas, sin que aquéllas alteren las jurisdicciones locales" (art. $41 \mathrm{CN}$ ). Por lo tanto, en virtud del dispositivo constitucional comentado, se produjo una nueva delegación de competencias a favor de la Nación, para que esta regule los "presupuestos mínimos de protección ambiental", que deben aplicarse al uso de los recursos naturales pero sin despojar a las provincias del dominio que les corresponde sobre aquellos (Sabsay, 1997, 786). Conforme la interpretación pacífica de la doctrina, al Estado Nacional le corresponde sancionar la legislación general, de base, que contenga presupuestos comunes de protección para todos los habitantes, mientras que a las provincias les corresponde complementar 
dicha legislación, incluyendo asuntos que incumban específicamente a cada una de ellas (Rosatti et al., 1994). En síntesis, en todo lo que no se refiera a presupuestos mínimos, las provincias conservan sus atribuciones para legislar en materia ambiental, ya que cada región necesita protección frente a problemas específicos (Gelli, 2001).

En ese marco de concertación federal se asientan los tres pilares normativos de la lucha contra el cambio climático: la Ley 24.295, de 1993, a través de la cual se ratificó la Convención Marco de las Naciones Unidas sobre Cambio Climático; la Ley 25.438, de 2001, por medio de la cual se ratificó el Protocolo de Kioto; y la Ley 25.675, de 2002, que establece la Política Ambiental Nacional. Ese plexo normativo es, a su vez, complementado con decretos y resoluciones que, en su conjunto, pretenden sentar las bases comunes de protección ambiental en todo el territorio nacional.

Como parte de la CMNUCC, Argentina asumió el compromiso de formular, aplicar, publicar y actualizar regularmente programas nacionales y regionales, que contengan medidas orientadas a mitigar el cambio climático, y en virtud de ello ya ha presentado tres comunicaciones nacionales contentivas de un inventario sobre los GEIs emitidos por el país y sus esfuerzos por reducir dichos niveles. Asimismo, en atención al compromiso asumido con motivo de la ratificación del Protocolo de Kioto, la modificación del sector energético ha sido uno de los ejes de trabajo para lograr la reducción de las emisiones de GEIS.

Como ya se mencionó, si bien Argentina no tiene un compromiso cuantificado de reducción de emisiones, debe realizar los esfuerzos necesarios para que su proceso de desarrollo implique la liberación de la menor cantidad posible de GEIs. En esa lógica, "durante el desarrollo de la 4ta. Conferencia (COP 4) realizada en Buenos Aires en el año 1998, el gobierno nacional anunció su intención de elaborar una meta de emisiones que Argentina se comprometería voluntariamente a cumplir durante el primer período de compromiso (2008-2012) que establece el Protocolo de Kioto"11. En ese contexto, un informe publicado por el Banco Mundial y realizado por la Fundación Bariloche muestra que las opciones de Argentina para la reducción de GEIS están concentradas en el sector energético (Secretaría de Energía de la Nación, 2004, 18) ${ }^{12}$.

11 Secretaría de Energía de la Nación, Descripción, desarrollo y perspectivas de las energías renovables en la Argentina y en el mundo, mayo de 2004, 18. Disponible en: https:// www.energia.gov.ar/contenidos/archivos/Reorganizacion/renovables/DescripcionDesarrolloyPerspectivas.pdf

12 Según lo expresado en el referido informe elaborado por la Fundación Bariloche, en 2004 las previsiones para 2015 mostraban que "un aumento en el uso de la energía hidroeléctrica es una de las alternativas más baratas para reducir emisiones. Sin embargo, los grandes incrementos en capacidad generadora pueden ser difíciles de implementar debido a inquietudes sociales y medioambientales asociadas con los desarrollos hidroeléctricos masivos. La energía 
Por su parte, la Política Ambiental Nacional (Ley 25.675) -que no constituye propiamente una política de cambio climático- establece los presupuestos mínimos para el logro de una gestión sustentable y adecuada del ambiente, la preservación y protección de la diversidad biológica y la implementación del desarrollo sustentable (art. 1); y a pesar de que no manifiesta en forma expresa una vinculación con las fuentes de energía renovable, todo el texto de la norma refleja la importancia de promover el desarrollo sustentable, el cual se basa en el desarrollo conjunto del medio ambiente, la sociedad y la economía, sin que la primacía de uno de esos factores afecte a los otros.

En igual sentido, con relación a la normativa dictada en materia de cambio climático, el Decreto 481/2000 del Poder Ejecutivo Nacional aprueba el memorando de entendimiento entre Argentina y el Banco Internacional de Reconstrucción y Fomento (BIRF) para la participación del país en el Fondo Prototipo Carbono, destinado a financiar proyectos para reducir las emisiones de GEIs, de acuerdo a lo establecido con relación a los Mecanismos para un Desarrollo Limpio (MDL), contenido en el Protocolo de Kioto. Es decir que este Fondo puede ser utilizado para implementar proyectos de energía limpia (renovable) en países en vías de desarrollo, como lo es Argentina. De hecho, según lo informa la CEPAL, en diciembre de 2010 se habían presentado 42 proyectos de MDL, dos de los cuales se encontraban en proceso de evaluación y consistían en la promoción de energías renovables: un proyecto trataba sobre energía geotérmica y el otro versaba sobre la sustitución de gas natural por biomasa (CEPAL, 2014, 210). En la misma línea, el Fondo Argentino del Carbono, creado por Decreto 1070/2005, tiene como objetivo promover y facilitar las inversiones y el desarrollo tecnológico en el marco de los Mecanismos para un Desarrollo Limpio.

A su vez, también relacionado con el vínculo entre cambio climático y energía renovable, la Resolución 1076/2001 de la Secretaría de Desarrollo Sustentable de la Nación crea el "Programa Nacional de Biocombustibles relacionado con la problemática del cambio climático", tendiente a implementar medidas y políticas que favorezcan el uso de biocombustibles como forma de proteger el medio ambiente reduciendo las emisiones de gases de efecto invernadero.

De forma concordante, la Disposición 166/2001 de la Subsecretaría de Ordenamiento y Política Ambiental dispuso la creación del Programa Na-

eólica es atractiva y tiene un vasto potencial técnico. Sin embargo, el potencial de esta energía se encuentra limitado por barreras de acceso a la trasmisión, un suministro intermitente y altos costos para los emprendimientos en pequeña escala. La energía hidroeléctrica y eólica juntas podrían reducir un total de emisiones de alrededor del 8\% hacia el 2015. Asimismo, una mayor eficiencia energética del sector residencial podría reducir un $2 \%$ del total de emisiones argentinas para ese período, así como podría lograrse una reducción del $1.5 \%$ en el sector transportista" (Secretaría de Energía de la Nación. Descripción, desarrollo y perspectivas de las energías renovables en la Argentina y en el mundo, cit., 18). 
cional de Energías y Combustibles Alternativos y Sustentables, con el fin de promover tecnologías y procedimientos para el desarrollo de energías limpias que contribuyan a la reducción de las emisiones de dióxido de carbono.

Finalmente, cabe señalar que, continuando con la línea trazada desde hace algunas décadas, el actual gobierno argentino creó, mediante Decreto Presidencial 891/16, del 25 de julio de 2016, el Gabinete Nacional sobre Cambio Climático, cuya función es la articulación de políticas en dicha materia y la generación de conciencia social sobre su relevancia (art. 1). Asimismo, la normativa establece que la nueva institución funcionará en el ámbito de la Jefatura de Gabinete y estará integrada por los titulares de los ministerios de Energía y Minería, Producción, Agroindustria, Transporte, Ambiente y Desarrollo Sustentable, Desarrollo Social, Relaciones Exteriores y Culto, Educación y Deportes, Ciencia, Tecnología e Innovación Productiva, del Interior, Obras Públicas y Vivienda, Hacienda y Finanzas, y de Cultura (art. 2). La composición del Gabinete, en el que participan casi todos los sectores del gobierno, muestra que el problema del cambio climático involucra no solo cuestiones ambientales, sino también sociales y económicas.

\subsection{La importancia de las fuentes renovables en la política energética argentina}

Más allá de la retórica gubernamental en cuanto a la conveniencia y utilidad de promover el uso de energías renovables para lograr el desarrollo sustentable, la sanción, en el año 2014, de la Ley 27.007 para la promoción de la fractura hidráulica (fracking) muestra que el destino energético de Argentina continúa fuertemente ligado al uso de fósiles (Freier y Schaj, 2016). A pesar de ello, vale destacar la labor normativa y política que se viene desarrollando para promover el aumento de la presencia de energía verde en la matriz nacional.

Desde una perspectiva histórica, en 1998 Argentina sancionó la Ley 25.019, por medio de la cual creó el Régimen Nacional de Energía Eólica y Solar destinado a promover el uso de ambas fuentes, a través de tres mecanismos: el estímulo a la investigación, la aplicación de un régimen impositivo diferenciado y la creación de un fondo fiduciario. Sin embargo, el proyecto no prosperó porque la crisis financiera que se desató a fines de 2001 y la necesidad de subsidiar la energía para mitigar los efectos de la crisis socioeconómica relegaron el tema en la agenda política nacional.

Posteriormente, en el año 2006 se sancionó la Ley 26.190, que creó el "Régimen de Fomento Nacional para el uso de fuentes renovables de energía destinada a la producción de energía eléctrica”, el cual vino a complementar la legislación anterior. Inserta en otro contexto social, político y económico nacional, la nueva ley renovó el interés en la temática incluyendo una mayor variedad de fuentes: energía eólica, solar, geotérmica, mareomotriz, hidráulica, biomasa y biogás. Asimismo, entendiendo que el éxito del proyecto dependía 
en gran medida del uso de las tecnologías adecuadas, el foco también estuvo puesto en el área de investigación y desarrollo, estableciéndose un plazo de 10 años para lograr que el $8 \%$ del consumo energético se sustentara en fuentes renovables.

En 2009 se reglamentó la ley a través del Decreto 562/09, pero dos problemas afectaron su implementación. Por un lado, el precio fijo garantizado fue insuficiente para atraer inversiones privadas (Rotaeche, 2014, 107), y por otro lado, los instrumentos de promoción económica y la devolución impositiva son mencionados como las principales razones del bajo rendimiento del sector de energía renovable en Argentina (Guzowski y Recalde, 2008, 3595).

Posteriormente, con el objetivo de cumplimentar la meta del $8 \%$ de energías renovables en la matriz energética, el gobierno creó el programa de licitación "Generación eléctrica a partir de fuentes renovables" (GENREN), el cual estuvo destinado a licitar, por 15 años, la compra de energía eléctrica de fuentes renovables y fue implementado por la empresa Energía Argentina S.A. (ENARSA) en dos etapas: GENREN I (2009) y GENREN II (2010). Este programa priorizó los proyectos nacionales, por lo que, al tiempo que se buscaba promover el sector económico nacional, se descartaba la participación de compañías con experiencia en mercados caracterizados por el uso de fuentes renovables.

Finalmente, en 2015, ante el insuficiente crecimiento del uso de las energías renovables, se sancionó la Ley 27.191, la cual buscó darle impulso al sector para alcanzar el $20 \%$ de contribución en la red eléctrica nacional hasta el 31 de diciembre de 2025. Algunos de los instrumentos económicos previstos para promover el uso de las fuentes alternativas son el Fondo para el Desarrollo de Energías Renovables (FODER), diferentes beneficios impositivos para los inversores y la prioridad de acceso a financiación pública a través del FODER para los proyectos con mayor componente nacional.

A modo de síntesis de lo expuesto sobre el panorama argentino, se puede afirmar que el entramado normativo y político reseñado muestra la estrecha vinculación que el Estado reconoce entre el cambio climático y el sector energético. De hecho, ha creado un sistema de normas e instituciones que buscan adaptar el sistema energético, procurando el desarrollo equilibrado de la economía, la sociedad y el medio ambiente.

Sin embargo, puesto que Argentina no posee ningún régimen específico de cambio climático, se advierte que su implementación resultaría adecuada para reforzar la promoción de la energía verde, en un contexto que muestra que el gobierno, a la par de fomentar el uso de fuentes renovables, también apoya la explotación de las fuentes tradicionales ${ }^{13}$.

13 Como se mencionó, la sanción de la Ley 27.007, en 2014, promueve la explotación de fósiles mediante la técnica de la fractura hidráulica. Además de ello, en materia nuclear, Argentina tiene tres plantas nucleares en funcionamiento (la Central Nuclear Juan Perón -ex Atucha I-, la Central Nuclear Embalse, y la Central Nuclear Néstor Kirchner -ex Atucha II-), 
4. LA RELACIÓN ENTRE MEDIO AMBIENTE Y ENERGÍA

EN LA REPÚBLICA FEDERATIVA DE BRASIL

Brasil, por ser un Estado federal, se enfrenta a la necesidad de delimitar la esfera de competencia de la Unión, los estados, los municipios y el Distrito Federal para legislar sobre el uso de los recursos naturales. En tal sentido, la Constitución de Brasil establece, por un lado, competencias exclusivas de la Unión (art. 22), y por otro, competencias concurrentes entre aquella, los estados y los municipios (art. 24).

A la Unión, entendida como el conjunto indisoluble de los estados, municipios y el Distrito Federal (art. 1), le corresponde legislar de manera exclusiva (arts. 22, inc. 4, 12 y $26 \mathrm{CN}$ ) sobre aguas, yacimientos, minas, recursos minerales, actividades nucleares y energía. Por su parte, es competencia concurrente de los cuatro estamentos de gobierno (art. 24, incs. 6, 7 y 8) legislar sobre flora, caza, pesca, fauna, conservación de la naturaleza, defensa del suelo, recursos naturales, protección del medio ambiente, control de la polución, protección del patrimonio turístico y paisajístico, y responsabilidad por daño ambiental (Monteiro de Rezende, 2013, 41). A partir de allí, puesto que muchas áreas se superponen (p. ej., la regulación de los recursos hídricos, conforme el art. 22, y la regulación de los recursos naturales, conforme el art. 24), las mayores dificultades interpretativas residen en la separación de los espacios reservados a los legisladores nacional y estadual, en el ámbito de la competencia concurrente en materia ambiental (Monteiro de Rezende, 2013, 42).

Partiendo de la premisa de que la competencia para legislar en materia ambiental es concurrente, corresponde a la Unión dictar normas generales; a los Estados, suplir lo que la legislación federal no regula y ejercer competencia legislativa plena cuando exista un vacío legal; y a los Municipios, legislar sobre los vacíos dejados por la legislación federal y estadual (Silva Antunes da Souza y Buzaglo Dantas, 2014) ${ }^{14}$.

En este contexto, Silva y Buzaglo explican que normas generales son aquellas que trazan parámetros genéricos, aplicables a todo el territorio nacional, dejando las especificaciones destinadas a adaptar a las particula-

no obstante lo cual en el año 2009, con motivo de la sanción de la Ley 26.566 sobre Actividad Nuclear, el Congreso declaró de interés nacional la construcción de una cuarta central nuclear (FREIER, 2016a).

14 En ese sentido se pronuncian los artículos 24 y 30 de la Constitución de Brasil. Artículo 24: "Compete a la Unión, a los Estados y al Distrito Federal legislar concurrentemente sobre: [...] 1. ${ }^{\circ}$ En el ámbito de la legislación concurrente, la competencia de la Unión se limitará a establecer normas generales; $2 .^{\circ}$ La competencia de la Unión para legislar sobre normas generales no excluye la competencia suplementaria de los Estados; $3{ }^{\circ}$ No existiendo la ley federal sobre aspectos generales, los Estados ejercerán la competencia legislativa plena, para atender a sus peculiaridades...". Artículo 30: "Compete a los Municipios: 1. legislar sobre asuntos de interés local; 2. suplementar la legislación federal y estatal en lo que cupiese...". 
ridades regionales y locales para la legislación supletoria de los estados y municipios (Silva Antunes da Souza y Buzaglo Dantas, 2014, 201). Por lo tanto, la competencia en materia ambiental es concurrente y sigue una lógica de especificación que involucra a todos los estamentos de gobierno.

Dentro de tal marco de concertación, si bien se puede observar una prolífica actividad regulatoria en materia ambiental (Silva Antunes da Souza y Buzaglo Dantas, 2014, 197), propiamente sobre cambio climático el sistema normativo consta de tres regulaciones: el Decreto 2.652/98, que promulga la CMNUCC y establece que su contenido resulta enteramente obligatorio dentro del país; el Decreto 5.445/05, que promulga el Protocolo de Kioto, estableciendo también la plena obligatoriedad de sus disposiciones; y la Ley 12.187/09, que instituye una Política Nacional sobre Cambio Climático, la cual fue posteriormente reglamentada mediante Decreto 7.390/10. A su vez, el sistema normativo descrito es complementado con un Plan Nacional y un conjunto de planes sectoriales de mitigación y adaptación al cambio climático ${ }^{15}$ (Ministério da Ciência, Tecnología e Inovação, 2016, 28).

Como consecuencia de haber ratificado la CMNUCC y el Protocolo de Kioto, Brasil presentó tres comunicaciones nacionales informando a la autoridad de aplicación de la Convención sus esfuerzos por reducir las emisiones de GEIs. Si bien, por ser un Estado no-Anexo I, carece de un compromiso cuantificado, está sin embargo obligado a desarrollar y actualizar periódicamente inventarios nacionales de emisiones antrópicas de GEIs no regulados por el Protocolo de Montreal, y a presentar una descripción general de las medidas previstas o adoptadas para implementar la Convención (Ministério da Ciência, Tecnología e Inovação, 2016, 21).

En ese contexto, el uso de energía renovable forma parte de la estrategia para la promoción del desarrollo sustentable, y es considerado uno de los componentes de los planes gubernamentales para la mitigación y el control del cambio climático. Sin embargo, curiosamente no constituye una herramienta utilizada por la Ley 12.187/09 para luchar contra la transformación del clima, lo cual plantea el interrogante de por qué el contenido de la ley no se corresponde con el de las políticas públicas.

15 Dicho conjunto de políticas públicas incluye el "Plan de acción para la prevención y control de la desforestación en la Región Amazónica", el "Plan de acción para la prevención y control de la desforestación y quema en El Cerrado", el "Plan decenal de expansión de energía", el "Plan sectorial de mitigación y adaptación al cambio climático para la consolidación de una economía de baja emisión de carbono en la agricultura", el "Plan sectorial de reducción de emisiones en la siderurgia", el "Plan sectorial de mitigación y adaptación al cambio climático para la consolidación de una economía de baja emisión de carbono en la industria de transformación", el "Plan de mitigación y adaptación al cambio climático en la minería de baja emisión de carbono", el "Plan sectorial de transporte y movilidad urbana para la mitigación y adaptación al cambio climático", y el "Plan sectorial de salud para la mitigación y adaptación al cambio climático". 
Como se señaló más arriba, en 1998 y en 2005 Brasil promulgó las respectivas leyes que tornan obligatorio el cumplimiento interno del contenido de la CMNUCC y el Protocolo de Kioto. De esa manera, el Estado asumió, también a nivel nacional, el compromiso de hacer esfuerzos por luchar contra el cambio climático y reducir las emisiones de GEIs. Ahora bien, ninguno de dichos textos se pronuncia en cuanto a la forma de cumplir tales objetivos, dejando, por tanto, abierta a la discrecionalidad nacional la definición de las estrategias. Es decir que, a efectos de reducir las emisiones de GEIs, Brasil puede elegir los sectores y mecanismos que considere más apropiados, incluyendo, o no, al sector energético.

En ese sentido, corresponde destacar, sin embargo, que tanto el Plan Nacional sobre Cambio Climático, del año 2008, como el Plan Decenal de Expansión Energética (que se actualiza anualmente desde 2007) centran su atención en la producción de energía renovable como estrategia contra el cambio climático.

El Plan Nacional sobre Cambio Climático (2008) se asienta sobre el entendimiento de que el cambio climático, más allá del diferente grado de responsabilidad que le cabe a cada país en cuanto al origen del problema, es un fenómeno que afecta a todos por igual. Por ende, a través del Plan Nacional, Brasil busca encontrar un camino a fin de que el esfuerzo para su mitigación sea efectivo y constituya una garantía para el bienestar de sus ciudadanos (Comitê Interministerial sobre Mudança do Clima, 2008, 7). En ese sentido, el Plan Nacional se ocupa del abordaje de diferentes áreas, cuyo funcionamiento implica la emisión de GEIs, a fin de elaborar estrategias que permitan reducir tales emanaciones. Las oportunidades de mitigación las encuentra en los sectores de energía, transporte, edificaciones, industria, agricultura, silvicultura y residuos.

En lo que respecta al sector energía, el objetivo es mejorar la eficiencia de la oferta y distribución, la sustitución de combustibles con alto contenido de carbono por otros con uno menor o por combustibles de fuentes renovables, y la captación y almacenamiento de carbono (Comitê Interministerial sobre Mudança do Clima, 2008, 28).

Dado que Brasil afirma tener una de las matrices energéticas más limpias del mundo gracias a sus grandes reservas acuíferas con las que produce energía hidroeléctrica, al uso de alcohol para el transporte, y al uso del bagazo de la caña de azúcar y el carbón vegetal para la industria (Comitê Interministerial sobre Mudança do Clima, 2008, 22), las emisiones de $\mathrm{CO}_{2}$ provenientes de la quema de combustibles fósiles son relativamente bajas en comparación con las de otros Estados, y la mayor cantidad de ellas tiene su origen en los cambios en el uso de la tierra, principalmente por la transformación de bosques en áreas agropecuarias (Comitê Interministerial sobre Mudança do Clima, 2008, 22). 
No obstante, la estrategia brasilera, implementada a través del Plan Nacional, apunta a aumentar aún más el porcentaje de renovables y energías limpias, para lo cual ha comenzado a trabajar con el fin de lograr la expansión de la generación hidroeléctrica, ha desarrollado un programa de incentivo a las fuentes alternativas de energía (Proinfa) y ha implementado licitaciones para la compra de energía de fuentes alternativas. Al mismo tiempo, como resultado de la preocupación por reducir los GEIs, sumada a la necesidad de velar por la seguridad energética, también ha incluido en su estrategia la expansión de la energía nuclear, considerara energía limpia. Por otro lado, los proyectos contemplados por el Plan Nacional incluyen la expansión de la energía solar fotovoltaica, la utilización de residuos urbanos para fines energéticos, el desarrollo de una política industrial para equipamientos eficientes y tecnologías renovables, la expansión de la producción de biocombustibles (etanol y biodiesel) y el uso energético de biomasa.

En sintonía con el Plan Nacional, que incluye al sector energético como una de las áreas en las cuales se puede trabajar para mitigar el cambio climático, el gobierno desarrolló también una estrategia específica en materia de energía. Así, se puso en marcha el Plan Decenal de Expansión Energética (PDE), que tiene como principal objetivo la incorporación de acciones que tiendan a lograr un escenario de bajas emisiones de $\mathrm{CO}_{2}$ en lo relativo a la producción y el uso de energía, considerando como aspectos clave la expansión de la oferta hidroeléctrica y de las fuentes renovables, principalmente en lo referente a centrales eólicas, pequeñas centrales hidroeléctricas y bioeléctricas, expansión de la oferta de biocombustibles y energía solar, e incremento de la eficiencia energética (Ministério da Ciência, Tecnología e Inovação, 2016, 31).

El PDE se actualiza anualmente y "presenta resultados de estudios prospectivos de demanda y oferta de energía, y de proyectos relacionados, como plantas de generación de electricidad, líneas de transmisión, refinerías de petróleo, bloques de exploración de petróleo, gasoductos, oleoductos, e instalaciones de producción de bioenergía" (Ministério de Minas e Energia, 2014). La última versión, el PDE 2024, indica que el balance energético espera alcanzar en 2024 el 45,2\% de participación de fuentes renovables en la matriz energética nacional, contribuyendo así a la generación del $86 \%$ de la oferta de electricidad. Asimismo, el mencionado Plan Decenal prevé, en el mismo horizonte temporal, el aumento de la producción de energía eólica del $2 \%$ al $8 \%$ y la diversificación a través del incremento de otras fuentes renovables, tales como los combustibles líquidos (Ministério de Minas e Energia, 2015, v-vi).

Luego de la incorporación a nivel interno de la cMNUCC y el Protocolo de Kioto, y tras la implementación de los planes -nacional y sectorial-que contemplan modificaciones en el sector energético como estrategia para reducir las emisiones de dióxido de carbono, el estado avanzó en la construcción de 
una Política Nacional de Cambio Climático, la cual fue implementada por Ley 12.187/09, y reglamentada mediante Decreto 7.390/10.

Puesto que el compromiso de reducción de emisiones asumido por Brasil a través del Protocolo de Kioto no es cuantificado (por ser Parte no-Anexo I), tal autolimitación fue fijada mediante Ley 12.187, cuyo artículo 12 establece que para alcanzar los objetivos de la Política Nacional sobre Cambio Climático el país adoptará, como compromiso voluntario, acciones para reducir entre el $36,1 \%$ y el $38,9 \%$ de sus emisiones de GeIs hasta 2020 .

Para tal fin, se establecen objetivos, directrices y una serie de instrumentos (arts. 4 a 7). Sin embargo, y a diferencia de la línea marcada por los planes nacional y sectorial, la Política sobre Cambio Climático no aborda, y de hecho excluye expresamente, el tema de la promoción de las energías renovables como estrategia para reducir las emisiones de dióxido de carbono.

En la redacción original de la ley, los artículos 4 (inc. 3) y 10 planteaban el estímulo al desarrollo y uso de tecnologías limpias, y la paulatina sustitución de la energía de origen fósil por otra de fuentes renovables, todo ello con miras a la protección del medio ambiente. Sin embargo, el Poder Ejecutivo ordenó el veto de ambos dispositivos normativos, argumentando que Brasil ya contempla el uso de energías renovables a través de legislación específica, y que el avance en el sentido de abandonar el uso de combustibles fósiles podría poner en riesgo la seguridad energética nacional ${ }^{16}$.

Un año más tarde, en diciembre de 2010, el gobierno aprobó el Decreto 7.390 que reglamenta los artículos 6, 11 y 12 de la Ley 12.187. En esta nueva reglamentación asoma el tema de la energía renovable vinculada al cambio climático, toda vez que el artículo 3 menciona al Plan Decenal de Expansión Energética como un instrumento para la mitigación del cambio climático. Si bien el PDE no se focaliza exclusivamente en energías renovables, sino que contempla un plan integral de abastecimiento energético que incluye también los combustibles fósiles y la energía nuclear, vale destacar-como se mencionó más arriba- que lo orienta la idea de lograr un escenario de bajas emisiones de $\mathrm{CO}_{2}$ en lo relativo a la producción y el uso de energía. Asimismo, el artículo 6 , párrafo $1 .^{\circ}$, inciso III, menciona que a los fines de cumplir con el compromiso asumido voluntariamente se procurará la expansión de la oferta hidroeléctrica, de la energía de fuentes alternativas renovables (principalmente centrales eólicas, pequeñas centrales hidroeléctricas y bioelectricidad) y de los biocombustibles.

16 Mensaje de veto del Poder Ejecutivo (Mensaje n. $\left.{ }^{\circ} 1.123 / 09\right)$. Disponible en: http:// www.planalto.gov.br/ccivil_03/_ato2007-2010/2009/Msg/vEP-1123-09.htm 


\subsection{La importancia de las fuentes renovables en la política energética brasilera}

Si bien Brasil proclama con orgullo la alta participación de la energía hidráulica en su matriz nacional, la oferta interna de energía se compone de un 37,3\% de petróleo y un 13,7\% de gas (Ministério de Minas e Energia, 2016, 4), y el descubrimiento, en 2007, de la primera reserva petrolífera "supergigante" -superior a 10 billones de barriles- colocó a esa nación entre los principales productores de energía en el mundo (Freier, 2016b, 166).

Sin embargo, más allá de la relevancia de los combustibles fósiles, Brasil ha instalado en los últimos años el discurso de la "diplomacia verde". En sintonía con esas ideas, en el año 2002 sancionó la Ley 10.438, mediante la cual creó el Programa de Incentivos para Recursos Alternativos de Energía (PROINFA) focalizado en el uso de biomasa, proyectos hidroeléctricos de baja escala y energía eólica, aplicando el sistema tarifario feed-in tariff (FIT $)^{17}$, el cual fue criticado por ser costoso, ineficiente y no competitivo en el sector energético.

Asimismo, el Banco Nacional de Desarrollo Económico y Social (BNDES) ofrece financiación para la instalación de plantas o parques de energía renovable, con tasas de interés muy bajas, pero para acceder a dichas líneas de crédito el PROINFA requiere que al menos un $60 \%$ del proyecto contenga piezas nacionales. De lo contrario, cuando la empresa generadora utiliza otra fuente de financiación diferente al BNDES, es posible importar todas las piezas necesarias. Sin embargo, las conexiones a la red de transmisión, el establecimiento de un sistema de transporte, el montaje e ingeniería civil adecuado para torres y los aerogeneradores son responsabilidad de los productores, lo que torna la energía eólica un 20-30\% más cara que en otros países que utilizan este recurso a gran escala.

En el año 2004 se sancionó la Ley 10.848, la cual también incluyó la energía solar, y reemplazó el sistema FIT por las licitaciones públicas, con el objetivo de garantizar el precio más bajo para el consumidor final y un horizonte a más largo plazo para los productores. En cuanto a las variadas fuentes de energía renovable, se planificó que compitan unas con otras para crear un mercado más competitivo; y, para lograr un abastecimiento de electricidad confiable, se obligó a las distribuidoras a contratar el cien por ciento de la demanda prevista para su mercado (Dutra y Szklo 2006, 861). En ese esquema de provisión de energía, la de origen eólico ha mantenido

17 El sistema feed-in tariff es "un instrumento normativo que impulsa el desarrollo de las ERNC, mediante el establecimiento de una tarifa especial, premio o sobre precio, por unidad de energía eléctrica inyectada a la red por unidad de generación ERNC (Energías Renovables No Convencionales). Es decir, interviene el precio que es recibido por el generador ERNC, obteniendo este actor claridad sobre el precio mínimo que le será pagado por concepto de electricidad" (Central Energía, 2010). 
la prioridad, quedando exenta del pago de Impuesto a la Circulación de Manufacturas y Servicios (ICMS) y del 75\% del Impuesto a la Rentabilidad (Rotaeche 2014,80-81). La primera subasta exclusiva de energía eólica tuvo lugar en diciembre de 2009, y los programas de licitación han mostrado ser capaces de reducir los precios de la energía drásticamente, encontrándose Brasil entre los países con menor precio de la energía derivada de fuentes renovables (Rotaeche, 2014, 75).

De lo dicho se puede concluir que en Brasil tanto el desarrollo de la energía renovable como el fomento de la lucha contra el cambio climático ocupan lugares destacados en la agenda política nacional. Sin embargo, el punto de convergencia entre ambas áreas está subordinado a la satisfacción de las necesidades energéticas nacionales, vinculadas al progreso económico del país.

\section{REFLEXIONES FINALES}

Retomando los interrogantes planteados en el inicio, y a efectos de dar un cierre al análisis, a continuación se reflexiona sobre la prevalencia del desarrollo sustentable o de la seguridad energética, como valor orientador de las políticas nacionales. Asimismo, teniendo en cuenta el contexto más amplio del Mercosur, del cual Argentina y Brasil forman parte, también se observa el rol de la institución regional en la definición de las estructuras normativas domésticas. Finalmente, se intenta arrojar luz sobre la existencia o la posible emergencia de estándares comunes que faciliten una futura integración regional en materia de energías renovables.

En primer lugar, Argentina se encuentra formalmente comprometida, tanto a nivel nacional como internacional, con la protección del medio ambiente. En ese sentido, el entramado normativo y político reseñado muestra la estrecha vinculación que el Estado reconoce entre el cambio climático y el sector energético, y la consecuente necesidad de la correlación de ambos sectores a fin de lograr la reducción de las emisiones de gases de efecto invernadero. Más allá de las dificultades concretas para avanzar en la complementación práctica de las dos áreas, presumiblemente debido a la presión de las empresas que manejan el mercado de los combustibles fósiles, Argentina ha creado un sistema de normas que proclama la necesidad de adaptar el sistema energético, procurando el desarrollo equilibrado de la economía, la sociedad y el medio ambiente.

Si bien no posee, a la fecha, ningún régimen de cambio climático, su implementación serviría para reforzar la promoción de la energía verde. En ese sentido, destacando la preocupación del Estado por satisfacer el abastecimiento energético, esencial para el crecimiento económico y social, el discurso nacional parece sugerir que la voluntad es lograr la seguridad energética a través de una matriz verde que garantice el desarrollo sustentable. No puede desconocerse, sin embargo, que continúa el apoyo del gobierno a 
la explotación de las fuentes tradicionales (energía de origen fósil y nuclear), lo cual ciertamente se vincula con la incapacidad actual para producir suficiente cantidad de energía renovable, y con el lobby ejercido por las empresas petroleras que se niegan a perder su lugar en el mercado.

Con relación a Brasil, aunque dicho Estado reconoce la utilidad de las energías alternativas renovables para reducir las emisiones de $\mathrm{CO}_{2}$, la Política Nacional sostiene que la prioridad es el logro del abastecimiento energético seguro (seguridad energética). Es decir que, aun cuando la protección del medio ambiente es un tema importante que ocupa un lugar en la agenda política, su importancia es relativa, ya que debe armonizarse con otros valores relevantes para la política y la seguridad energéticas, necesarias para el desarrollo del país.

Por ello, la política brasilera contra el cambio climático se focaliza con mayor intensidad en temas como la lucha contra la deforestación, la quema de bosques y los cambios en el uso de la tierra, los cuales tienen gran vinculación con el calentamiento global, pero no conducen a la redefinición de conceptos que puedan afectar la provisión interna de energía. En este sentido debe ser mencionado también que, aunque el régimen de cambio climático de Brasil no incluye el tema de las fuentes renovables para no afectar la seguridad energética nacional, los planes para reducir las emisiones de dióxido de carbono van de la mano con los proyectos del Estado para extender su base de energía limpia.

La lectura hecha hasta este punto sugiere que, a nivel del discurso, Brasil ha declarado su inclinación por la seguridad energética por sobre el desarrollo sustentable, mientras que Argentina parece colocar ambos valores en pie de igualdad. Sin embargo, a nivel empírico se observa claramente que ambos Estados procuran, por igual, su abastecimiento energético, aun a costa del medio ambiente, si bien promueven el aumento del uso de energías renovables para modificar sus matrices energéticas a mediano o largo plazo.

En cuanto a la influencia que ejerce el marco regional en la configuración de las políticas nacionales, como ya se mencionó, el Mercosur funciona como un foro de comunicación y negociación para orientaciones políticas y comerciales diferentes, a pesar de que no existe, por el momento, un régimen de cambio climático regional que comprometa a los Estados a coordinar sus políticas para promover el uso de fuentes renovables de energía.

En ese sentido, a pesar de las diferencias ideológicas entre ambos Estados, se pueden observar algunos elementos, surgidos a partir de un incipiente proceso de difusión ${ }^{18}$ originado en el contexto regional, que permiten

18 La difusión es un proceso por el cual la información sobre innovaciones políticas o normativas es comunicada en el sistema internacional y esas políticas son luego adoptadas voluntaria y unilateralmente por un creciente número de Estados a lo largo del tiempo. La difusión se refiere a la propagación internacional de flujos de innovación política más que a presión política 
descartar la rotunda fragmentación legal y sugieren la posibilidad de lograr un gradual acercamiento normativo y político, para una futura cooperación energética sustentable. En ese sentido, los principales elementos que hablan del gradual surgimiento de estándares energéticos sustentables comunes incluyen la creación de fondos públicos de financiación para el desarrollo del sector de las energías renovables, la implementación de procesos de licitación para maximizar la eficiencia de la producción de energía verde, el estímulo a la industria nacional mediante el otorgamiento de prioridad en el acceso a financiación a las empresas con mayor componente doméstico, y la implementación de programas para la formación de capacidades en materia de producción de energía renovable.

\section{REFERENCIAS}

Busch, P.-O.; Jörgens, H. (2012). Europeanization through diffusion? Renewable energy policies and alternative sources for European convergence. En: Morata y Solorio Sandoval (eds.). European Energy Policy: An Environmental Approach, Cheltenham, UK: Edward Elgar, 66-82.

Central Energía - Central de Información y Discusión de energía en Chile. Feed-in Tariff. 13/07/10. Disponible en: http://www.centralenergia.cl/2010/07/13/feed-in-tariff/

Cepal. (2014). La economía del cambio climático en la Argentina. Chile: Naciones Unidas, CEPAL.

Comitê Interministerial sobre Mudança do Clima. (2008). Plano Nacional sobre Mudança do Clima-PNMC-Brasil. Brasilia. Disponible en: http://www.mma.gov.br/estruturas/ smcq_climaticas/_arquivos/plano_nacional_mudanca_clima.pdf

Dutra, R. M. y Szklo, A. S. (2006). A Energia Eólica no Brasil: Proinfa e o Novo Modelo do Setor Elétrico. Anais do XI Congresso Brasileiro de Energia - CBE. Rio de Janeiro. Vol. II, 842-868.

ElKind, J. (2009). Energy Security - Call for a Broader Agenda. En: PAscual, C. y Elkind, J. (eds.). Energy Security, Economics, Politics, Strategies, and Implications, Washington: Brookings Institution Press, 119-148.

FreIer, A. (2016a). La situación de la cooperación energética entre Argentina y Brasil en el área de la energía renovable: ¿integración, difusión o fragmentación? Relaciones Internacionales. Vol. 25, n. ${ }^{\circ}$ 51. Disponible en: http://revistas.unlp.edu.ar/RRII-IRI/ article/view/2951

FreIER, A. (2016b). Integración energética regional en Mercosur. Los casos de Argentina y Brasil en el área de la energía renovable. En: Guzowski (comp.). Políticas de pro-

o a decisiones colectivas dentro de las instituciones. A nivel micro, los procesos de difusión involucran mecanismos de aprendizaje político, copia, emulación mimética y competencia política y económica (BusCH y JöRGENs, 2012, 70). 
moción de las Energías Renovables. Experiencias en América del Sur. Bahía Blanca: Ediuns, 153-192.

Freier, A. y Schaj, G. (2016). La fractura hidráulica en Argentina: los cambios en el concepto de territorialidad y la emergencia de nuevos regímenes de soberanía. Revista Enfoques, Universidad Central de Chile. Vol. 14, n. ${ }^{\circ} 25,59-81$.

Gelli, M. A. (2001). Constitución de la Nación Argentina. Comentada y concordada. Buenos Aires: La Ley.

Guzowski, C. y Recalde, M. (2008). Renewable Energy in Argentina. Energy Policy Analysis and Perspectives. International Journal of Hydrogen Energy. Vol. 33, n. ${ }^{\circ}$ 13, 3592-3595.

Ministério da Ciência, Tecnología e Inovação. (2016). Sumario Executivo. $3 .^{a}$ Comunicação Nacional do Brasil ã Convenção-Quadro das Nações Unidas sobre Mudança do Clima. Brasilia. Disponible en: http://sirene.mcti.gov.br/documents/1686653/1706739/ MCTI _ TCN _ SUMARIO+EXECUTIVO _port.pdf/7aad0f1d-332b-45b4-9fda-88e9efb049fd

Ministério de Minas e Energia. (2015). Plano Decenal de Expansão de Energia 2024 - Sumário. Brasília: MME/EPE. Disponible en: http://www.epe.gov.br/pdee/forms/ epeestudo.aspx

Ministério de Minas e Energia. (2016). Resenha Energética Brasileira 2016 - Resultados 2015. Disponible en: http://www.aben.com.br/Arquivos/456/456.pdf

Ministério de Minas e Energia. (2014). Expansión de Energía en Brasil-2023. Oportunidades de Inversiones. Disponible en: http://www.mme.gov.br/documents/10584/3596336/03++Expansi\%C3\%B3n+de+Energ\%C3\%ADa+en+Brasil+Oportunidad+de+Inversiones+ +(PDF)/b3309e1f-6657-46b8-aecf-64d3311903cf;jsessionid=746214DDE00CF35D18615 B00C7AA0E01.srv155?version $=1.1$

Monteiro de Rezende, R. (2013). Normas gerais revisitadas: A competência legislativa em matéria ambiental. Textos para Discussão 121. Núcleo de Estudos e Pesquisas do Senado. Disponible en: https://www12.senado.leg.br/publicacoes/estudos-legislativos/ tipos-de-estudos/textos-para-discussao/td-121-normas-gerais-revisitadas-a-competencialegislativa-em-materia-ambiental

Nonna, S.; Waitzman, N. y Dentone, J. M. (2011). Ambiente y residuos peligrosos. Buenos Aires: Estudio.

Rosatti, H.; Barra, R.; García Lema, A.; Masnatta, H.; Mosset Iturraspe, J.; Paixão, E. y Quiroga Lavié, H. (1994). La Reforma de la Constitución. Explicada por miembros de la comisión de redacción. Buenos Aires: Rubinzal-Culzoni.

Rotaeche, L. M. (2014). Energías renovables en Argentina. Una propuesta para su desarrollo. Buenos Aires: Dunken.

SABSAY, D. A. (1997). El nuevo artículo 41 de la Constitución Nacional y la distribución de competencias Nación-provincias. Doctrina Judicial. Año III, n. ${ }^{\circ}$ 28, 783-787.

Secretaría de Ambiente y Desarrollo Sustentable de la Nación. (2015). Tercera Comunicación Nacional de la República Argentina a la Convención Marco de las Na- 
ciones Unidas sobre Cambio Climático. Buenos Aires. Disponible en: http://unfccc. int/resource/docs/natc/argnc3s.pdf

Secretaría de Energía de la Nación. (2004). Descripción, desarrollo y perspectivas de las energías renovables en la Argentina y en el mundo. Disponible en: https://www. energia.gov.ar/contenidos/archivos/Reorganizacion/renovables/DescripcionDesarrolloyPerspectivas.pdf.

Secretaría Mercosur. (2006). Medio ambiente en el Mercosur. Relevamiento $n .^{\circ}$ 001/06. Montevideo: Secretaría Mercosur.

Silva Antunes de Souza, M. C. y Buzaglo Dantas, M. (2014). Competência legislativa em matéria ambiental no Brasil e a análise das decisões do Supremo Tribunal Federal. En: Hoanat, Rezende y Saleme (orgs.). Direito Ambiental V: XXIII Congresso Nacional do CONPEDI. A humanização do Direito e a horizontalização da justiça no século XXI. Florianópolis: CONPEDI. Vol. 1, 194-221.

Silva Gilli, R. (2004). Medio ambiente en el Mercosur. Série Grandes Eventos - Meio Ambiente. Brasília: Escola Superior do Ministério Público da União (ESMPU), 2004, 49-62.

Stuhdreher, A. (2012). Construcción participativa del regionalismo estratégico: ¿hacia una agenda medioambiental externa del Mercosur? Revista Brasilera de Política Internacional. ‥ ${ }^{\circ} 55$ (1), 194-210.

Urrutia Silva, O. (2010). El Régimen Jurídico Internacional del Cambio Climático después del "Acuerdo de Copenhague. Revista de Derecho de la Pontificia Universidad Católica de Valparaíso, 597-633.

Yergin, D. (2006). Ensuring Energy Security. Foreign Affairs. Vol. 85, n. o 2, 69-82.

\section{Normativa}

Constitución de la República Federativa de Brasil. Diário Oficial da União, Brasilia, 5 de octubre de 1988.

Convención Marco de Naciones Unidas sobre el Cambio Climático.

Decreto 1070/2005. Fondo Argentino del Carbono. Boletín Oficial, Buenos Aires, 5 de septiembre de 2005.

Decreto 481/2000. Reducción de Gases con efecto invernadero. Boletín Oficial, Buenos Aires, 13 de junio de 2000.

Decreto 562/2009. Energía Eléctrica. Boletín Oficial, Buenos Aires, 15 de mayo de 2009.

Decreto 7.390/2010. Diário Oficial da União, Brasilia, 10 de diciembre de 2010.

Disposición 166/2001. Programa Nacional de Energías y Combustibles Alternativos y Sustentables. Boletín Oficial, Buenos Aires, 24 de octubre de 2001.

Ley 10.438. Diário Oficial da União, Brasilia, 29 de abril de 2002.

Ley 10.848. Diário Oficial da União, Brasilia, 16 de marzo de 2004. 
Ley 12.187. Diário Oficial da União, Brasilia, 29 de diciembre de 2009.

Ley 24.430. Constitución Nacional Argentina. Boletín Oficial, Buenos Aires, 10 de enero de 1995 .

Ley 25.019. Régimen Nacional de Energía Eólica y Solar. Boletín Oficial, Buenos Aires, 26 de octubre de 1998

Ley 25.675. Política Ambiental Nacional. Boletín Oficial, Buenos Aires, 28 de noviembre de 2002 .

Ley 26.190. Régimen de Fomento Nacional para el Uso de Fuentes Renovables de Energía Destinada a la Producción de Energía Eléctrica. Boletín Oficial, Buenos Aires, 27 de diciembre de 2006.

Ley 27.191. Régimen de Fomento Nacional para el Uso de Fuentes Renovables de Energía destinada a la Producción de Energía Eléctrica. Modificación. Boletín Oficial, Buenos Aires, 15 de octubre de 2015.

Mensaje de veto del Poder Ejecutivo n. ${ }^{\circ}$ 1.123/09. Disponible en: http://www.planalto.gov. br/ccivil_03/_ato2007-2010/2009/Msg/veP-1123-09.htm

Protocolo de Kioto.

Resolución 1076/2001. Programa Nacional de Biocombustibles. Boletín Oficial, Buenos Aires, 29 de agosto de 2001.

Tratado de Asunción de 1991. 